\title{
Potential Methods For Improving Pedestal Temperatures and Fusion Performance *
}

\author{
G.W. Hammett ${ }^{1}$, W. Dorland ${ }^{2}$, M.A. Beer ${ }^{1}$, M. Kotschenreuther ${ }^{3}$ \\ 1 Princeton Plasma Physics Laboratory \\ 2 Univ. of Maryland Inst. for Plasma Research \\ 3 Univ. of Texas Inst for Fusion Studies
}

(October 6, 1999)

\begin{abstract}
The physics of the tokamak edge is very complicated, and the scaling of the H-mode transport barrier pedestal has significant uncertainties. Evidence from the largest tokamaks appears to support a model in which the H-mode pedestal width scales linearly with the poloidal gyroradius and the gradient scales with ideal MHD ballooning limits. However, there appears to be significant variability in the data from different tokamaks, including observations on DIII-D that indicate a regime where the pedestal is in second stability and the width is independent of poloidal gyroradius, which would give a more favorable scaling to reactor scales. An important question is the role of the bootstrap current in the pedestal, and another is how far can the improvements in edge stability be pushed with higher triangularity and elongation. Even with the more pessimistic model, where the pedestal width is proportional to the poloidal gyroradius, the results presented here suggest that pedestal temperatures, and thus the fusion performance, may be significantly improved by designs with stronger plasma shaping (higher triangularity and elongation), moderate density peaking, and higher magnetic field (and thus reduced size), such as in ARIES-RS, FIRE, and some of the new ITER-RC designs.
\end{abstract}

\section{THE SENSITIVITY OF FUSION PERFORMANCE TO TRANSPORT}

It is well known that the fusion gain $Q$ is a sensitive function of the confinement time $\tau_{E}$, particularly near ignition. Making standard energy balance assumptions, $P=P_{\alpha}+P_{\text {aux }}=3 n T V / \tau_{E}$, with the alpha power $P_{\alpha} \propto n^{2} T^{2}$, ignoring bremsstrahlung, and assuming fixed fuel dilution, leads to the result $Q=5 /\left[\left(n T \tau_{E}\right)_{\infty} /\left(n T \tau_{E}\right)-1\right]$, where $\left(n T \tau_{E}\right)_{\infty}$ is the value of $n T \tau_{E}$ at which ignition occurs. Normalizing $\tau_{E}$ to a Goldston-like scaling, $\tau_{E} \propto H / P^{1 / 2}$ leads to $Q=5 /\left(K^{2} / H^{2}-1\right)$, where $K$ is a coefficient that depends on the size and shape etc. of the device. Just a $30 \%$ drop in the confinement multiplier $H$ can reduce $Q$ from ignition $(Q=\infty)$ to $Q=5$, and a $50 \%$ drop (such as if an H-mode is not achieved) would yield $Q=1.7$. Thus it is important to try to understand and reduce the uncertainties in $\tau_{E}$, so that the amount of design margin that is needed in a particular experiment to study high $Q$ is reduced. Conversely though, relatively moderate improvements in the confinement time can lead

\footnotetext{
*Contributed paper for workshop on Opportunities and Directions in Fusion Energy Science for the Next Decade, Snowmass, CO July 1999. www.pppl.gov/snowmass/details.html
} 
to large increases in the fusion power, which may allow one to reduce the size and cost of a fusion power plant significantly (assuming that corresponding improvements in the MHD beta limits and the engineering heat load constraints can be achieved). Such possibilities are being actively explored in advanced tokamak experiments in the U.S. and elsewhere. [A more revolutionary approach that might further improve tokamak and other power plant concepts is the use of liquid walls. This was discussed at Snowmass by Kotschenreuther, Zakharov, Woolley, and others, and won't be discussed further here.]

Because of these issues, the BPX design team caried out a careful study of the ignition margin and the sensitivity of that design to uncertainties in transport ${ }^{1}$. One of the main concerns raised about the original ITER-EDA design in 1995-1997 was that the uncertainties in extrapolating the global H-mode database were larger than were being assumed ${ }^{2,3}$. There were also concerns that confinement might be lower than the original extrapolations because of the difficulty of achieving at large scales some of the factors that are often correlated with improved confinement in present devices. These include high $T_{i} / T_{e}$, density peaking, high rotational shear, dilution by beams and impurities, and operating away from limits in the Greenwald density, beta, and the H-mode power threshold. The FESAC 1997 report $^{2}$, and in particular its physics basis appendix, contains a detailed discussion of these and other technical concerns. Of course the uncertainties go in both directions, and the original ITER-EDA design may have worked just fine. Since the time of the FESAC 1997 report, there has been progress in understanding some of these issues better, and the statistical conditioning of the global database has improved with the addition of new data, though there are still systematic variations between tokamaks and between different parameter regimes in the database that need further study, and significant uncertainties remain.

In this paper we will focus on a related transport issue, that of the scaling of the edge pedestal temperature in the H-mode transport barrier. Central temperature profiles and fusion performance are often empirically correlated with edge conditions and high edge temperatures (or pedestal temperatures in the case of H-modes). There is a range of transport models presently under study with differing degrees of stiffness, or sensitivity to the edge boundary conditions (see Ref.[4] and references therein by Waltz, Kinsey, Bateman and others). Gyrokinetic particle simulations have found a lower $\chi$ than the original gyrofluid simulations (the discrepancy is reduced by recent modifications of the gyrofluid closures to improve the treatment of neoclassical flows). Modifying the IFS-PPPL transport model to approximate the lower gyrokinetic $\chi$ still gives predictions that are fairly sensitive to the assumed pedestal temperature and/or the achievable density, though there are a number of uncertainties that need further investigation ${ }^{4}$. But the main point of this paper is to explore potential directions for significantly increasing the pedestal temperature, which is favorable to varying degrees in all transport models.

\section{EMPIRICAL AND THEORETICAL PEDESTAL SCALINGS.}

A standard picture of the H-mode that has developed over the last decade is in terms of ExB shear suppression of turbulence, (building on the work of Ref.[5] and many others, see the review by Burrell ${ }^{6}$ ). Kotschenreuther and Dorland 7,3 used this "conventional-wisdom" picture to develop a scaling for the H-mode pedestal along the following lines (see Fig. 1). [Perkins et.al. ${ }^{8}$ independently derived a similar scaling at the same conference, and used it as part of a possible explanation of the Greenwald density limit. Actually, the essential features of this scaling, that the width is proportional to the poloidal gyroradius and the gradient is set by ideal MHD, appeared first in experimental papers. See Fig. 1(b) of 
Ref.[9], and other papers cited below.] Turbulence is assumed to be suppressed where the ExB shearing rate $\partial\left(c E_{r} / B\right) / \partial r$ (using a simple circular form) is larger than the linear growth rate $\gamma \propto \omega_{* p}$, with the maximum $\gamma=G v_{t} / L_{p}$, where $G$ depends on various dimensionless parameters. Assuming radial force balance neglecting neoclassical flows, $\partial p / \partial r \sim e n E_{r}$, this gives the requirement

$$
\frac{\partial}{\partial r}\left(\frac{c}{e B n} \frac{\partial p}{\partial r}\right)>\gamma=\frac{v_{t}}{L_{p}} G
$$

Assuming $\partial / \partial r \sim 1 / \Delta$, and $L_{p} \sim \Delta$, this gives the pedestal width $\Delta \sim \rho / G$. Assuming that the maximum pressure gradient in this pedestal region is given by the ideal MHD ballooning limit, $\alpha=$ $R q^{2} \partial \beta / \partial r=\alpha(\hat{s}, \kappa, \delta, \ldots)$ (where the maximum normalized pressure gradient $\alpha$ depends on various dimensionless factors), then $\beta$ at the top of the pedestal is given by

$$
\beta_{p e d}=\Delta \partial \beta / \partial r \approx \rho \alpha /\left(G R q^{2}\right)
$$

This can then be solved to find the pedestal temperature $T_{p e d}$ as a function of the pedestal density $n_{\text {ped }}$ and other parameters.

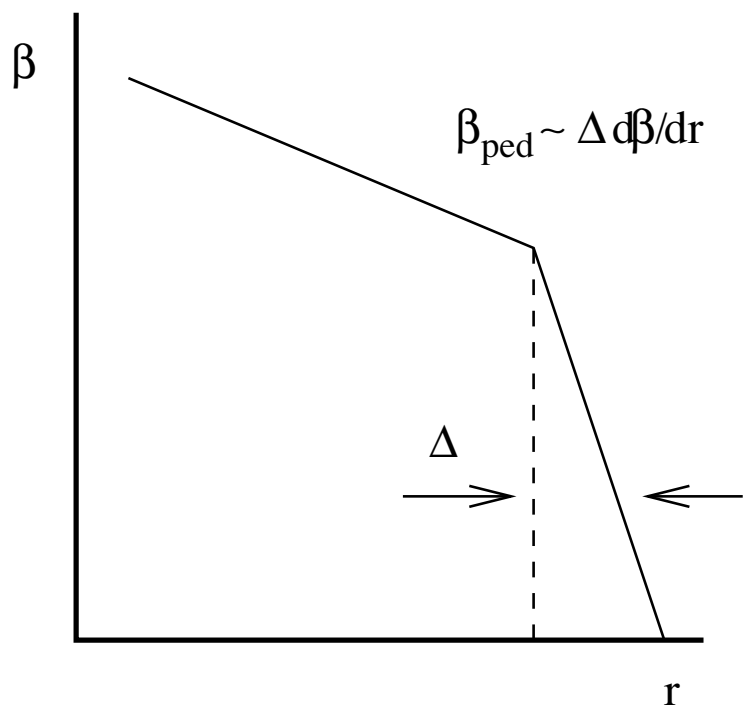

FIG. 1. Conceptual picture of an ELMy H-mode plasma. The turbulence is suppressed in an edge boundary layer of width $\Delta$. If a scaling for the maximum pressure gradient $d \beta / d r$ is given, then $\beta_{p e d}$ at the top of the pedestal can calculated.

A number of papers by the JT-60U team ${ }^{9-12}$ (some of which predated and motivated the scaling papers $^{7,3,8}$ ) and by the JET team ${ }^{13}$, provide experimental evidence that (1) the width of the H-mode pedestal scales with the banana width, $\Delta \propto \sqrt{r / R} \rho_{\theta}$ (consistent with the above scalings with a particular choice for $G$ ), and (2) the gradient in the H-mode pedestal region scales with the ideal MHD ballooning limit. [Because the pedestal width is relatively narrow, it can be difficult to accurately measure the width $\Delta$ and the gradient $\partial \beta / \partial r$ separately. But what matters most to provide a boundary condition for core transport models is the pedestal height $\beta_{\text {ped }}=\Delta \partial \beta / \partial r$, which is easier to measure. For example, Ref.[13] focuses on the scaling of the pedestal height $\beta_{\text {ped }}$, which it finds is consistent with these combined assumptions for the pedestal width and the pedestal gradient.] 
Increased plasma shaping has a favorable effect on edge MHD pressure limits and H-mode performance $^{11,12}$. The Troyon beta limit can be written as

$$
\beta_{\text {Troyon }}=C_{T} \frac{I}{a B}=C_{T} \frac{5 a}{q_{95} R} \frac{\left[1+\kappa^{2}\left(1+2 \delta^{2}-1.2 \delta^{3}\right]\right.}{2} \frac{(1.17-0.65 a / R)}{\left(1-(a / R)^{2}\right)^{2}} \equiv C_{T} \frac{5 a}{q_{95} R} U_{q}
$$

(using a version of the Uckan formula ${ }^{14}$ relating $q$ to current, field, and shape), which shows how $\beta$ improves with elongation $\kappa$ and triangularity $\delta$ ( $\kappa$ and $\delta$ evaluated at the $95 \%$ flux surface) at fixed $q$. If the global volume averaged $\beta$ is this sensitive to the triangularity, which is large only near the plasma edge and falls rapidly towards the magnetic axis, then the local pressure gradient is probably much more sensitive to the triangularity. A rough fit to the empirical triangularity dependence found in Refs. $[11,12]$ gives $\alpha \propto\left[1+\kappa_{x}^{2}\left(1+10 \delta_{x}^{2}\right)\right]$. In this paper we will use a slightly weaker scaling $\alpha \propto\left[1+\kappa^{2}\left(1+10 \delta^{2}\right)\right]$, $\left(\kappa \equiv \kappa_{95} \approx 0.914 \kappa_{x}\right.$ and $\delta \equiv \delta_{95} \approx 0.85 \delta_{x}$ typically).

Combining this expression for $\alpha$ with the above scaling of the pedestal width proportional to banana width, which we roughly approximate for general geometry as $\Delta \propto \sqrt{\epsilon} \rho_{\theta} \propto \rho q \sqrt{R / r} / \kappa$ (we are measuring the width at the outer midplane, and the factor of $\kappa$ very roughly accounts for the increase in the local $B_{\theta}$ with elongation at fixed $q$ ), gives

$$
T_{p e d}=C_{0}\left(\frac{n_{G r}}{n_{p e d}}\right)^{2}\left[\frac{1+\kappa^{2}\left(1+10 \delta^{2}\right)}{1+\kappa^{2}\left(1+2 \delta^{2}-1.2 \delta^{3}\right)} \frac{\left(1-(a / R)^{2}\right)^{2}}{(1.17-0.65 a / R)}\right]^{2} \frac{A_{i} R}{\kappa^{2} a}
$$

where $n_{G r}=I_{p} /\left(\pi a^{2}\right) 10^{20} / \mathrm{m}^{3}$ is the Greenwald density, with plasma current $I_{p}$ in MA and minor radius $a$ in $m, n_{\text {ped }}$ is the pedestal density, $\kappa$ and $\delta$ are the elongation and triangularity, $A_{i}$ is the average hydrogenic ion mass, and $R$ is the major radius. $C_{0}$ is a constant we will determine by normalizing to a JET case ${ }^{15}$ (the first line of Table I). [A more accurate approximation to the general geometry $\Delta$ of Ref.[10], which uses $\bar{B}_{\theta}=\int d l_{p} B_{\theta} / \int d l_{p}$ in the definition of $\rho_{\theta}$, results in multiplying Eq. (3) by $(1+\kappa)^{2} /\left(4 U_{q}^{2} \kappa\right)$, where $U_{q}$ is defined in Eq. (2). The results are intermediate between the third and second-from-last columns of Table I.] Clearly a number of rough analytic approximations were made in deriving this scaling, and more detailed numerical calculations and comparisons with existing experiments need to be done.

As a check on Eq. (3), one can scale from another JET paper. Eq. 5 from Ref.[13] incorporates the scaling for the pedestal height into an offset-nonlinear form for the confinement time, $\tau_{E}=\tau_{E, \text { core }}+W_{\text {ped }} / P$. This can be used to work out a scaling for the pedestal temperature, and the result is

$$
T_{\text {ped }}=\left(\frac{n_{G r}}{n_{\text {ped }}}\right)^{2} \frac{A_{i}}{2 \kappa}\left(\frac{3 a}{R}\right)^{2} 0.22 \mathrm{keV}
$$

This is similar to Eq. (3), but lacks the shape scalings because Eq. 5 from Ref.[13] was based on plasmas of a fixed shape (approximately like the ITER-96 design), and was not intended to be extrapolated to other shapes. For this standard shape, it extrapolates to a pedestal temperature for the ITER-96 design of about $0.12 \mathrm{keV}$, about $40 \%$ lower than the result in Table I, which uses Eq. (3) and is extrapolating from a different JET case. More work is needed to understand the scatter in the data.

Other theoretical models of the pedestal have also been proposed. A model based on peeling instabilities ${ }^{18}$ predicts $\Delta \propto \rho^{2 / 3} R^{1 / 3}$, a bit weaker than the above result $\Delta \propto \rho$. One can also get this scaling if the 
microinstabilities of interest are driven by bad curvature, so the linear growth rate $\gamma \sim \sqrt{\omega_{* p} \omega_{d}}$, and the $\max \gamma \sim v_{t} / \sqrt{\Delta R}$. Following similar steps as in Eq. (1), this leads to a pedestal width $\Delta \propto \rho^{2 / 3} R^{1 / 3}$. Instead of using the fastest growing mode, if one assumes that ExB suppression of low- $k$ modes is relevant (low- $k$ modes are slower growing but may have large mixing length transport estimates), and that the lowest $k$ that needs to be considered is such that the local growth rate $\gamma \sim v_{t} /(q R)$, the connection rate to the good curvature side, then the pedestal width scaling is $\Delta \propto \sqrt{\rho R q}$. This gives an even more optimistic scaling, and is also shown in Table I. In the simulations by Waltz et.al. ${ }^{19}$, it was better to use the fastest growing mode in the shearing rate criterion, so the $\Delta \propto \rho$ or $\Delta \propto \rho^{2 / 3} R^{1 / 3}$ assumptions are probably better. $\left[\Delta \propto \rho^{2 / 3} R^{1 / 3}\right.$ would give predictions intermediate between the last column and third-from last column of Table I.] Edge turbulence is very complicated, but detailed nonlinear edge simulations, such as being done by Scott, et.al. at Garching, Drake et.al. at the Univ. of Maryland, and others, are making rapid progress and may soon give a firmer basis for choosing the right scaling.

\begin{tabular}{||l|r|r|r|r|r|r|r|r|c|c|c|c||}
\hline \hline & $\begin{array}{r}\mathrm{R} \\
\mathrm{m}\end{array}$ & $\begin{array}{r}\mathrm{a} \\
\mathrm{m}\end{array}$ & $\begin{array}{r}\mathrm{B} \\
\mathrm{T}\end{array}$ & $\begin{array}{r}I_{p} \\
\mathrm{MA}\end{array}$ & $\begin{array}{r}n_{\text {ped }} \\
10^{20} / \mathrm{m}^{3}\end{array}$ & $\frac{n_{\text {ped }}}{n_{\text {Gr }}}$ & $\frac{n_{\text {ped }}}{\langle n\rangle}$ & $\kappa_{95}$ & $\delta_{95}$ & $\begin{array}{c}T_{\text {ped }} \\
\mathrm{keV} \\
\text { if } \Delta \propto \rho_{\theta} \sqrt{\epsilon}\end{array}$ & $\begin{array}{c}T_{\text {ped }} \\
\mathrm{keV} \\
\text { if } 5 \delta^{2}\end{array}$ & $\begin{array}{c}T_{\text {ped }} \\
\mathrm{keV} \\
\text { if } \Delta \propto \sqrt{R q \rho}\end{array}$ \\
\hline JET-norm & 2.92 & 0.91 & 2.35 & 2.55 & 0.4 & 0.40 & $\sim 1$ & 1.61 & .17 & 2.1 & 2.1 & 2.1 \\
\hline ITER-96 & 8.14 & 2.80 & 5.68 & 21.0 & 1.3 & 1.52 & 1 & 1.60 & .24 & $0.20^{*}$ & $0.18^{*}$ & $1.5^{*}$ \\
lower $n_{\text {ped }}$ & 8.14 & 2.80 & 5.68 & 21.0 & 0.6 & 0.70 & .70 & 1.60 & .24 & $0.94^{*}$ & $0.83^{*}$ & $4.2^{*}$ \\
\hline ITER-HAM & 6.30 & 1.81 & 6.58 & 13.0 & 0.86 & 0.68 & .8 & 1.58 & .26 & 1.4 & 1.2 & 4.5 \\
\hline ITER-LAM & 6.45 & 2.33 & 4.25 & 17.0 & 0.64 & 0.64 & .8 & 1.70 & .43 & 2.0 & 1.2 & 5.5 \\
\hline Aries-RS & 5.52 & 1.38 & 7.98 & 11.3 & 1.4 & 0.74 & .67 & 1.70 & .50 & 3.4 & 1.9 & 7.7 \\
\hline FIRE & 2.0 & 0.53 & 10.0 & 6.44 & 3.6 & 0.48 & .80 & 1.77 & .40 & 4.8 & 3.0 & 6.7 \\
\hline \hline
\end{tabular}

Table I. Extrapolation of pedestal scalings from a JET discharge to various proposed devices. The third from last column uses Eq. (3), the scaling favored in papers from the two largest tokamaks, which assumes the pedestal width $\Delta \propto \rho_{\theta} \sqrt{\epsilon}$ with the gradient set by ideal MHD. The next to last column uses a modified version of Eq. (3), with the $10 \delta^{2}$ term in $\alpha$ reduced to $5 \delta^{2}$. The last column uses the more optimistic scaling $\Delta \propto \sqrt{R q \rho}$ (and the full $10 \delta^{2}$ term in $\alpha$ ). While there is significant variation between the models, they all predict that the pedestal temperature can be significantly improved by increasing the triangularity, elongation, and field (at fixed $q$, these also reduce the size and increase the Greenwald density) and by assuming that a modest amount of density peaking is achievable. [Some of the designs in Table I are evolving, so the parameters are approximate.]

While the results cited above from the two largest tokamaks support this model where the pedestal height is proportional to a banana width times the ideal MHD gradient, there are results from other tokamaks that appear to give contradictory evidence. Some of the initial JET papers found scalings of $T_{p e d}$ vs. $n_{\text {ped }}$ that were weaker than Eq. (3), more consistent with $\Delta \propto \rho^{2 / 3-1 / 2}$. Some of the earlier DIII-D papers said it was hard to distinguish between $\Delta \propto \rho_{\theta}^{2 / 3}$ and $\Delta \propto \beta_{\text {pol }}^{1 / 2} \rho_{\theta}^{0}$. But later experiments with a pumped divertor were able to break the correlation between $n_{p e d}$ and $I_{p}$, and found that the width scales with $R \beta_{\text {pol }}^{1 / 2}$, and the gradient exceeds the ideal MHD beta limit. Their analysis ${ }^{16,17}$ finds that because of the strong gradients in the pedestal, the bootstrap current can be large enough to lower the magnetic shear enough to get below the "nose" in the $\hat{s}-\alpha$ diagram and get into the second stability regime. This is an exciting possibility, since $\Delta \propto R$ gives a much more favorable scaling to large reactors, so it would seem important to reproduce this result on larger tokamaks. 
A recent paper by Sugihara et.al ${ }^{20}$ analyzes results from multiple tokamaks. They show results from several tokamaks that individually are consistent with a linear $\Delta \propto \rho$ scaling (at the ideal MHD gradient), but the different tokamaks have different coefficients and do not overlay when plotted together. In passing they mention that this may be evidence of some size scaling, but then they go on to give what we believe are more likely explanations. For example, the JT-60U data in their Fig. 2 is from the end of the ELM-free period, while the JET data in Fig. 2 is during ELMs, and JT-60U has reported that the pedestal width increases by a factor of 2-3 in the ELMy phase, which would bring it into line with the JET data. The C-MOD data is the lowest in their Fig. 2, but C-MOD does not see regular ELMs. They see a very interesting type of $\mathrm{H}$-mode they call EDA (Enhanced $D_{\alpha}$ ), and the physics that determines the pedestal in EDA could easily be significantly different than in ELMy H-modes. Sugihara et.al. also point out that the edge pressure gradient is sensitive to shape, and that detailed shape data was not yet available for all of their data.

Sugihara et. al. also make an interesting suggestion about the possible importance of the bootstrap current in the pedestal region, building on the DIII-D analysis ${ }^{16,17}$ mentioned above. Increasing the bootstrap current causes the pedestal magnetic shear to drop, which in the first stability regime will be unfavorable and reduce the beta limit. [If the bootstrap current can be made large enough and the shear small enough then one can get into second stability as DIII-D did.] Because the bootstrap current depends on collisionality, this can introduce hidden collisionality dependence into the $\beta$ limit that should be used in a pedestal model. This might explain why some data sets from JET (see Fig.3 of Ref.[20]) see a scaling weaker than the $T_{p e d} \propto 1 / n_{\text {ped }}^{2}$ predicted by $\Delta \propto \rho$ and $\alpha$ fixed (because the bootstrap current is changing as collisionality changes), while other JET data sets (see Ref.[13] and Figs. 1 and 2 of Ref.[20]) are consistent with $\Delta \propto \rho$ and $\alpha$ fixed (if they are from dimensionless scaling scans where $\beta$ and $\nu_{*}$ are held fixed during the scan). If so, the main assumptions of $\Delta \propto \rho$ and ideal MHD pressure limits used in earlier papers would still hold, but including the effect of bootstrap currents on the MHD beta limit would be a useful generalization. [Earlier scaling papers tended to use the standard dimensionless scaling framework where $\nu_{*}, \hat{s}, \beta$, etc., and thus the bootstrap current fraction, are held fixed (since present experiments can achieve reactor values of these) to focus on the $\rho_{*}$ scaling.] The bootstrap current needs to be included in future analysis to know quantitatively how important it is.

\section{DISCUSSION}

In summary, some of the new designs under consideration may be able to achieve significantly higher pedestal temperatures, which would be favorable for fusion performance. This is summarized in Table I and Eq. (3), which show that, even with the relatively pessimistic $\Delta \propto \rho$ scaling, it may be possible to achieve relatively high pedestal temperatures by going to high field, smaller size, stronger plasma shaping (higher elongation and triangularity), and moderate density peaking. Note that $n_{G r} \propto I_{p} / a^{2} \propto$ $B /(R q)\left[1+\kappa^{2}\left(1+2 \delta^{2}\right)\right]$ (approximately), so raising $B$ and reducing the size is very favorable.

To see the potential favorable impact of higher pedestal temperatures on fusion performance, see Ref.[4] (and references therein) for plots of $Q$ vs. $T_{p e d}$ from various transport models. Such $Q$ vs. $T_{p e d}$ plots should be done for the specific new designs under consideration in Table I, but these new designs are higher elongation and triangularity, and more work on the core transport models needs to be carried out to be confident of their scalings in those regimes. 
More work is needed to be more confident of these pedestal scalings, to understand how far the favorable influence of triangularity and elongation can be pushed, and to understand the role of the bootstrap current. Another important issue is whether moderate density peaking can be achieved. Note that a large value of central over volume-averaged density is not needed, we just need to reduce the edge pedestal density relative to the volume-averaged density, which might be achievable with even shallow pellet injection, though this also depends on the particle transport in the edge/pedestal region. There is also a limit on how low the density can be at the last closed field line for divertor operation. The preferred design developed over the last few years for ITER involves a radiative divertor that appears to be able to work with significantly lower densities than some of the earlier designs.

One caveat is that the increase in the pedestal temperature is correlated with an increase of energy per ELM dumped onto the divertor plates $^{17}$. It would useful to study possible methods of inducing more frequent ELMs (via pellets, etc.) or some other ELM control technique that might avoid reducing pedestal temperature. The C-MOD EDA mode may have advantages in this regard. Liquid protection on the divertor plates could also help.

Acknowledements: This work was supported by U.S. Department of Energy contracts DE-AC0276CH03073 and DE-FG05-80ET-53088, and DOE Grant DEFG02-93ER54197. We have had helpful conversations with Dr. Tom Osborne, Dr. Amanda Hubbard, and others.

\footnotetext{
${ }^{1}$ R.G. Goldston R.E. Waltz, G. Bateman, et.al. Fusion Tech. 21, 1076 (1992).

${ }^{2}$ See in particular the Physics-Basis Subpanel report in Appendix D.I of Panel Report To Fusion Energy Sciences Advisory Committee (FESAC) - "Review of the International Thermonuclear Experimental Reactor (ITER) Detailed Design Report", April 18, 1997, DOE/ER-0700. (wwwofe.er.doe.gov/morehtml/FESAC/ITER.Report.pdf \& wwwofe.er.doe.gov/more html/FESAC/Appendices.pdf).

${ }^{3}$ M. Kotschenreuther and W. Dorland, "Memorandum on Confinement Projections," Feb. 14, 1997 (w3.pppl.gov/ hammett/ark/1997/FESAC_ITER_Review_memo.pdf).

${ }^{4}$ A.M. Dimits, G. Bateman, M.A. Beer, et.al., "Comparisons and physics basis of tokamak transport models and turbulence simulations", UCRL-JC-135376 (August 1999), submitted to Phys. Plasmas (1999) (www.acl.lanl.gov/ GrandChal/Tok/papers/cyclone-b26.ps or w3.pppl.gov/ hammett/ark/1999/cyclone-b26.pdf).

${ }^{5}$ H. Biglari, P. Diamond, P.W. Terry, Phys. Fluids B 2, 1 (1990).

${ }^{6}$ K.H. Burrell, Phys. Plasmas 4, 1499 (1997).

${ }^{7}$ M. Kotschenreuther, W. Dorland, Q.P. Liu et.al., 16th IAEA Fusion Energy Conf. (Montreal, 1996).

${ }^{8}$ F.W. Perkins, D. Post, M. Rosenbluth, et.al., 16th IAEA Fusion Energy Conf. (Montreal, 1996).

${ }^{9}$ Y. Kamada, K. Ushigusa, O. Naito, et.al., Plasma Phys. Control. Fusion 36, A123 (1994).

10 T. Hatae, Y. Kamada, S. Ishida, et.al., Plasma Phys. Control Fusion 40, 1073 (1998)

${ }^{11}$ Yoshihiko Koide and the JT-60 Team, Phys. Plasmas 4, 1623 (1997).

${ }^{12}$ Y. Kamada, R. Yoshino, K. Ushigusa, et.al, 16th IAEA Fusion Energy Conf. (1996).

${ }^{13}$ J.G. Cordey and JET Team, 17th IAEA Fusion Energy Conf. (Yokohama, 1998)

${ }^{14}$ D.P. Stotler, W.T. Reiersen, G. Bateman, Comp. Phys. Comm. 81, 261 (1994).

${ }^{15}$ G. Janeschitz, A. Hubbard, Yu. Igitkhanov, et.al., 24th EPS Conf. on Contr. Fusion and Plasma Physics, 993 (1997).

16 T.H. Osborne, K.H. Burrell, R.J. Groebner, et.al., J. of Nucl. Mat., 266, 131 (1999).

17 T.H. Osborne, R.J. Groebner, L.L. Lao et.al., Plasma Phys. Contr. Fusion 40, 845 (1998)

${ }^{18}$ H.R. Wilson, J.W. Connor, A.R. Field, et.al., 17th IAEA Fusion Energy Conf. (1998).

${ }^{19}$ R.E. Waltz, , G.D. Kerbel, and J. Milovich, Physics of Plasmas 1, 2229 (1994).

${ }^{20}$ M. Sugihara, Yu. Igitkhanov, G. Janeschitz, et.al., 26th EPS Conf. http://epsppd.epfl.ch (1999).
} 